\title{
METODOLOGIA BIM APLICADA À GESTÃO DE ATIVOS
}

\author{
(1) ISEL: a39027@ alunos.isel.pt \\ (2) LNEC: mjoaofalcao@lnec.pt \\ (3) LNEC: pcouto@lnec.pt \\ (4) ISEL: joao.hormigo@edp.pt
}

Svetlana João $^{(1)}$, Maria João Falcão Silva ${ }^{(2)}$, Paula Couto ${ }^{(3)}$, João Hormigo ${ }^{(4)}$

\begin{abstract}
Resumo
A metodologia Building Information Modelling (BIM), encontra-se em franca expansão no setor de Arquitetura, Engenharia, Construção e Operação (AECO), englobando um conjunto de procedimentos que permitem melhorar a partilha da informação ao longo das diversas fases do processo construtivo, como por exemplo, maior produtividade, melhor cooperação interdisciplinar, automatização dos fluxos de trabalhos e otimização de prazos, minimização dos custos, diminuição dos erros de compatibilidade de projetos, etc. A Gestão de Ativos é uma área desafiante e de cada vez maior importância na sociedade moderna atual, na medida em que uma gestão eficiente e uma manutenção e operação eficazes de ativos (bens, equipamentos, entidades, infraestruturas, etc.) podem trazer inúmeros benefícios às organizações que os detêm. A evolução mundial e o crescimento económico potenciaram um aumento da necessidade de se conhecerem bem os ativos existentes, de forma a melhor controlá-los, recorrendo à análises holísticas dos processos da organização, priorizando e avaliando os objetivos fundamentais destas.

O trabalho desenvolvido tem como objetivo implementar ao caso estudo, uma Unidade Operativa Laboratorial de Ensaios Hidráulicos (UOLEH) a metodologia BIM na Gestão de Ativos. A referida unidade é modelada em BIM com a informação e detalhe necessários aos requisitos para a implementação de um sistema de Gestão de Ativos, com base nas Normas ISO 55000. No âmbito do estudo é analisado o estado atual das práticas de uma gestão de ativos nas instalações laboratoriais, por forma a identificar a posição da organização em relação aos requisitos das normas. Para finalizar, o estudo apresenta / propõe um método utilizando o "Construction Operations Building Information Exchange" (COBie) que demonstre as vantagens da metodologia BIM aplicada à Gestão de Ativos.
\end{abstract}

\section{Introdução}

Atualmente, as organizações têm como um dos seus principais objetivos a gestão cada vez mais eficiente e eficaz dos seus ativos, devido a inquietações geradas pelo crescimento cada vez mais complexo da economia [1]. 
Em 2008, foi publicada a primeira especificação para a "Gestão Otimizada de Ativos Físicos", pelo British Standards Institution (BSI), em colaboração com o Institute of Asset Management (IAM), a Publicly Available Specification (PAS) 55 2008, que se encontra dividida nas seguintes partes: i) PAS 55-1 2008 - Identifica os requisitos e especificações que têm de ser cumpridas de modo a otimizar a gestão de ativos físicos ao longo do seu ciclo de vida [2]; ii) PAS 55-2 2008 - Guia de orientações que possibilitam a aplicação dos requisitos presentes na PAS 55-1 2008 [3].

Atendendo à grande adesão e popularidade, foi proposto um projeto de transformação das especificações em normas, pela International Organization for Standardization (ISO), tendo surgido uma série de novas Normas ISO 55000 para a Gestão de Ativos, publicadas em 2014. Esta série de normas é dividida em três documentos: i) ISO 55000:2014 - Gestão de Ativos: Visão Geral, Princípios e Terminologia [4]; ii) ISO 55001:2014 - Sistemas de Gestão de Ativos - Requisitos [5]; iii) ISO 55002:2014 - Sistemas de Gestão de Ativos - Orientações [6].

O Building Information Modelling (BIM) é a gestão de informação durante todo o ciclo de vida de um ativo construído. Ele agrega valor ao apoiar a criação e a ligação de modelos compartilhados e dados estruturados de forma inteligente [7]. Conforme definido pelo National Institute for Building Science (NIBS), o BIM é uma representação digital das características físicas e funcionais de uma instalação. Um modelo de informação da construção é um recurso de conhecimento compartilhado para informações sobre uma instalação que forma uma base confiável para decisões durante seu ciclo de vida, definido como existindo desde a conceção inicial até à demolição [8].

A aplicação do BIM à gestão de ativos contribui para melhorias significativas numas organizações, tais como [9]: i) Esclarece como devem ser criados e compartilhados os dados e informações, declarando os requisitos para quem fornece os dados, e quais as verificações que precisam ser realizadas de forma a garantir que sejam atingidos os objetivos; ii) Aproxima pessoas, processos, informações e tecnologia; iii) Fornece uma única fonte de informação de dados dos ativos, para serem compartilhados durante a fase de projeto, construção e operação dos ativos. Com estas grandes vantagens, o BIM transformou-se num dos desenvolvimentos mais promissores no setor das indústrias de Arquitetura, Engenharia, Construção e Operação (AECO) [7].

\section{Gestão de Ativos}

A Gestão de Ativos é definida na especificação PAS 55 2008, como sendo, o conjunto das 'atividades sistemáticas e coordenadas, através das quais uma organização efetua uma gestão ótima e sustentável dos seus ativos e sistemas de ativos, seu desempenho, seus riscos e custos ao longo do ciclo de vida, por forma a atingir o plano estratégico' [2][3].

A gestão de ativos busca otimizar o custo, o risco e o desempenho durante todo o ciclo de vida do ativo, podendo ser um sistema ou apenas um ativo individual. $\mathrm{O}$ ciclo de vida de um ativo abrange desde a identificação inicial da necessidade do mesmo até o fim da sua vida útil, incluindo atividades como a conceção, o fabrico, a instalação e a exploração até ao seu fim [1]. Os ativos encontram-se divididos em diferentes tipos [10]: i) Ativos financeiros, que compreendem os lucros, os custos associados ao ciclo de vida, o valor dos ativos consoante o seu desempenho e as divisas; ii) Ativos humanos, que incluem a motivação, a comunicação, as responsabilidades, o conhecimento, a experiência, a competência e capacidade, a liderança e o 
trabalho em equipa; iii) Ativos de informação, que compreendem registos, desenhos, contratos, licenças, documentos legais e regulamentares e informação do nível de desempenho; iv) Ativos intangíveis, em que se inclui a reputação, as restrições morais e éticas e o impacto social; v) Ativos físicos, que compreendem os edifícios, os veículos e a qualidade do produto.

A gestão de ativos e a metodologia BIM devem beneficiar das potencialidades que existem da sua utilização conjunta, nomeadamente com as possibilidades de: i) Desenvolver novos ativos da organização no modelo BIM; ii) Agrupar os ativos existentes no modelo BIM; ou iii) Gerir e operar ativos existentes e / ou novos no BIM, de forma a atingir os objetivos determinados pela organização. Para melhor benefício destas vantagens o gestor de ativos deve implementar as informações de forma correta [11].

\section{Metodologia BIM}

O BIM é um dos desenvolvimentos promissores no setor AECO. De acordo com a tecnologia BIM, um determinado modelo virtual de um edifício é construído digitalmente, e após concluído, o modelo gerado contém geometrias precisas e dados relevantes para apoiar as atividades de construção, fabrico e aquisição necessárias para realizar o edifício [7].

Os projetos tradicionais dependiam em grande parte de desenhos técnicos bidimensionais, como plantas, alçados, cortes, etc. À medida que o ciclo de vida do projeto avança, essas informações tornam-se cada vez mais ricas em detalhes, até o ponto em que os dados do projeto são entregues a um cliente na conclusão da construção [1].

No entanto, os modelos BIM estendem-se muito para além de um simples aumento para as três principais dimensões espaciais (3D) (largura, altura e profundidade), com a inclusão de outras dimensões como: i) o tempo na quarta dimensão (4D); ii) o custo na quinta dimensão (5D); iii) a sustentabilidade na sexta dimensão (6D); e iv) a manutenção e gestão de ativos na sétima dimensão (7D). O modelo BIM, portanto, cobre mais do que apenas a geometria, cobrindo também relações espaciais, análises de luz, informações geográficas e quantidades e propriedades de componentes da construção (por exemplo, detalhes dos fabricantes) [8].

As dimensões do BIM são diferentes dos níveis de maturidade do BIM, referindo-se estes últimos à maneira como determinados tipos de dados são vinculados a um modelo de informações. Os níveis de maturidade são: i) Nível 0 - Sem BIM, Baixa Colaboração, 2D - uso de formatos 2D no Computer Aided Design (CAD) - baseia-se no processo tradicional de troca de informação da forma mais simples; ii) Nível 1 - Colaboração Parcial, 2D-3D - baseia-se no uso de formatos 2D no CAD para a apresentação de documentos e informações de equipamentos e o uso de formatos 3D para a conceção dos projetos; iii) Nível 2 - Colaboração Total, 4D-5D - distingue-se pelo trabalho colaborativo em que todas as partes usam os seus próprios modelos $3 \mathrm{D}$, mas não trabalham necessariamente num único modelo compartilhado; iv) Nível 3 - Integração Total, Open BIM, 6D - representa uma colaboração completa entre todas as disciplinas, por meio do uso de um único modelo de projeto compartilhado, que é mantido num repositório centralizado [13].

Relativamente ao nível de desenvolvimento (Level of Development - LOD), o Instituto Americano de Arquitetos (AIA) definiu cinco níveis, desde o LOD 100 até ao LOD 500, que são: i) LOD 100 - Modelo conceptual (Conceptual); ii) LOD 200 - Modelo de geometria aproximada (Approximate Geometry); iii) LOD 300 - Modelo de geometria mais precisa 
(Precise Geometry); iv) LOD 400 - Modelo de fabrico (Fabrication); e v) LOD 500 - Telas Finais (As-built) [14].

A Interoperabilidade retrata a necessidade de passar dados entre aplicações, de forma a permitir que vários tipos de especialistas e softwares contribuam para o trabalho em questão. É considerada uma das principais características do BIM, pois elimina a necessidade de replicar a entrada de dados que já foi gerada e facilita a fluidez dos fluxos de trabalho e automatização [7].

Sendo assim, este é o ponto chave do caso de estudo, pois, a partir da interoperabilidade do BIM é que será realizada a ligação entre a metodologia BIM e a Gestão de Ativos. O software BIM do caso de estudo é o Revit e o Plug-in (ligação de interoperabilidade) será o COBie (Construction Operations Building Information Exchange) [15]. O COBie corresponde a especificações relacionadas com informações de gestão de ativos de uma instalação. É um formato de dados para a publicação de um subconjunto de modelos de informações de construção (BIM) com foco no fornecimento de dados de ativos, distintos das informações geométricas [16].

\section{Caso de Estudo}

Pretende-se implementar a metodologia BIM à Gestão de Ativos de uma Unidade Operativa Laboratorial para Ensaios Hidráulicos (UOLEH), bem como avaliar se os objetivos traçados serão devidamente atingidos.

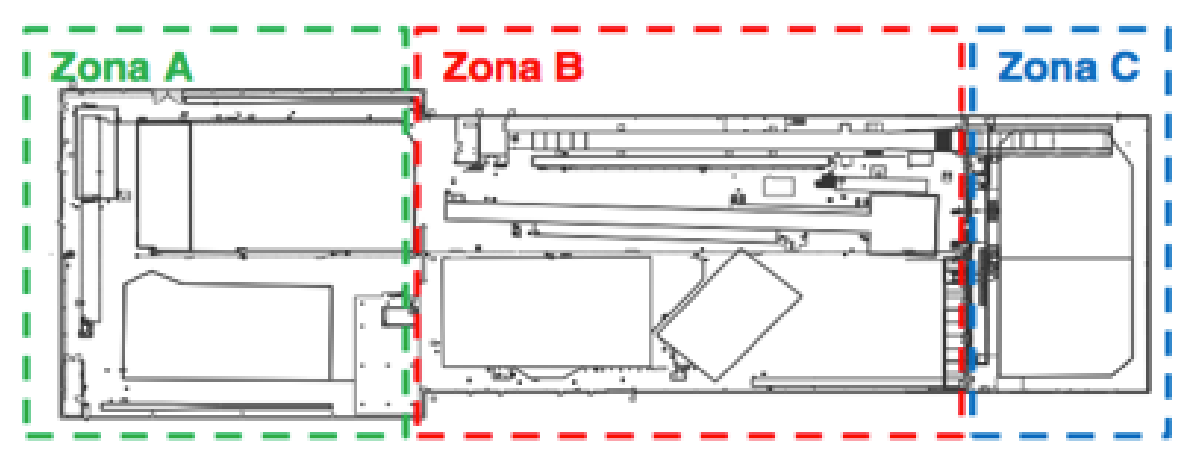

Figura 1: Planta da instalação da UOLEH, com as suas três zonas.

\subsection{Proposta de Modelo de Gestão de Ativos para o Caso de Estudo}

Para o desenvolvimento das atividades de gestão de ativos na UOLEH em estudo, foi elaborado um conjunto de processos devidamente identificados, de acordo com as normas ISO 55000 e o ciclo de gestão PDCA (Plan, Do, Check, Act / Action). O plano de Gestão de Ativos de acordo com o ciclo de Deming corresponde às etapas: i) Definição da estratégia e requisitos (Plan); ii) Plano de gestão de ativos (Do); iii) Avaliação de desempenho (Check); iv) Plano de ação (Act ou Action).

\subsection{Definição da estratégia e requisitos (Plan)}

A primeira etapa corresponde à definição da estratégia e dos requisitos da gestão de ativos da UOLEH. Nesta etapa definem-se os objetivos estratégicos, isto é, o que se pretende atingir com as atividades de gestão de ativos da UOLEH. A implementação dos objetivos estratégicos de 
gestão de ativos foi desencadeada pelas seguintes situações: i) envelhecimento das instalações; ii) aumento das exigências do serviço; iii) aumento de exigências económicas e ambientais; e iv) análise da relação de custo-eficiência.

Tabela 1: Definição dos objetivos estratégicos da UOLEH

\begin{tabular}{ll}
\hline Objetivos estratégicos & \multicolumn{1}{c}{ Descrição do objetivo } \\
\hline $\begin{array}{l}\text { Sustentabilidade social - Adequação da } \\
\text { interface com o utilizador }\end{array}$ & $\begin{array}{l}\text { Gerir uma instalação de ensaios hidráulicos, } \\
\text { pressupõe assegurar o cumprimento dos } \\
\text { ensaios com a máxima qualidade e } \\
\text { fiabilidade expectável. }\end{array}$ \\
& $\begin{array}{l}\text { Identificando que os ativos da instalação } \\
\text { são uma parte fundamental, devendo } \\
\text { preservar a sua integridade e avaliar as suas } \\
\text { capacidades para satisfação das } \\
\text { necessidades. }\end{array}$ \\
Sustentabilidade da gestão do serviço & $\begin{array}{l}\text { Agrupar um conjunto de indicadores } \\
\text { relativos à otimização e gestão eficiente dos } \\
\text { ativos, de forma garantir sustentabilidade } \\
\text { ambiental. }\end{array}$ \\
\hline Sustentabilidade ambiental &
\end{tabular}

\subsection{Plano de gestão de ativos (Do)}

Nesta segunda etapa agrupou-se um conjunto de informação para implementação do Plano de Gestão de Ativos, centralizado no Modelo Abrangente e Integrado de Gestão de Ativos Industriais (MAIGAI) [11] e nas atividades de manutenção na UOLEH.

Este modelo tem como finalidade aplicar a Norma ISO 55001 e propor uma metodologia aplicável a instalações de ensaios hidráulicos. Os elementos considerados como principais para o sistema de gestão de ativos na UOLEH são: i) Gestão do negócio (uma visão clara da instalação e das suas atividades, uma boa liderança, uma boa capacidade de comunicação); ii) Engenharia (conceito de impacto ambiental, conceito risco / fiabilidade); iii) Operação e manutenção (melhoria contínua, trabalho de equipa, fiabilidade); e iv) Gestor de ativos (pessoa responsável por verificar se estão a ser cumpridos os planos de gestão de ativos e a legislação regulamentar).

\subsection{Avaliação de desempenho (Check)}

Nesta etapa procede-se à monitorização da eficiência do sistema de gestão de ativos e do desempenho desta atividade na UOLEH, para que se possam identificar, por processos detalhados através da análise SWOT (Strengths - Forças, Weaknesses - Fraquezas, Opportunities - Oportunidades, Threats - Ameaças) e o Grau de Maturidade da gestão dos ativos, e implementar, posteriormente, melhorias e / ou correções onde forem necessárias (no Plano de Ação). Para a avaliação do grau de maturidade foram realizadas 29 perguntas para cada um dos 27 requisitos da ISO 55001, elaboradas pela IAM em 2014. O grau de maturidade que se obteve foi o nível "2" (Desenvolvimento). 


\subsection{Plano de ação (Act ou Action)}

No plano de ação deve-se trabalhar para que, na ocorrência de uma não conformidade ou um incidente nos ativos, na gestão de ativos ou no sistema de gestão de ativos na UOLEH, sejam realizadas determinadas ações como: i) tomar medidas para controlar e corrigir; ii) lidar com as consequências; iii) avaliar as necessidades de ações para eliminar as causas dos incidentes, de modo a que não se repita ou não ocorra; iv) determinar as causas da não conformidade ou incidente; e v) determinar a existência de não conformidades similares ou da suscetibilidade de virem a ocorrer.

\section{Modelação em BIM}

No âmbito do presente trabalho foram modeladas apenas as especialidades de Arquitetura (Figura 2) e Redes de Instalações (MEP) (Figura 3) da UOLEH (Figura 4), tendo a especialidade de estrutura sido excluída porque os seus componentes não precisam de manutenção frequente, sendo objeto por exemplo de inspeções regulares.

Para aplicação da metodologia BIM na Gestão de Ativos da UOLEH, foi escolhido o programa BIM - Revit 2018, criado pela Autodesk, e o Plug-in para ligação com a Gestão de Ativos foi o COBie. Primeiro, criou-se o modelo BIM de Arquitetura e MEP, de seguida adicionou-se toda a informação necessária, como por exemplo: equipamentos, tipos, localização, caraterísticas, entre outros. De seguida, exportou-se toda a informação para as folhas de cálculo (COBie). Deste modo, concretiza-se com maior facilidade a Gestão de Ativos da instalação UOLEH.

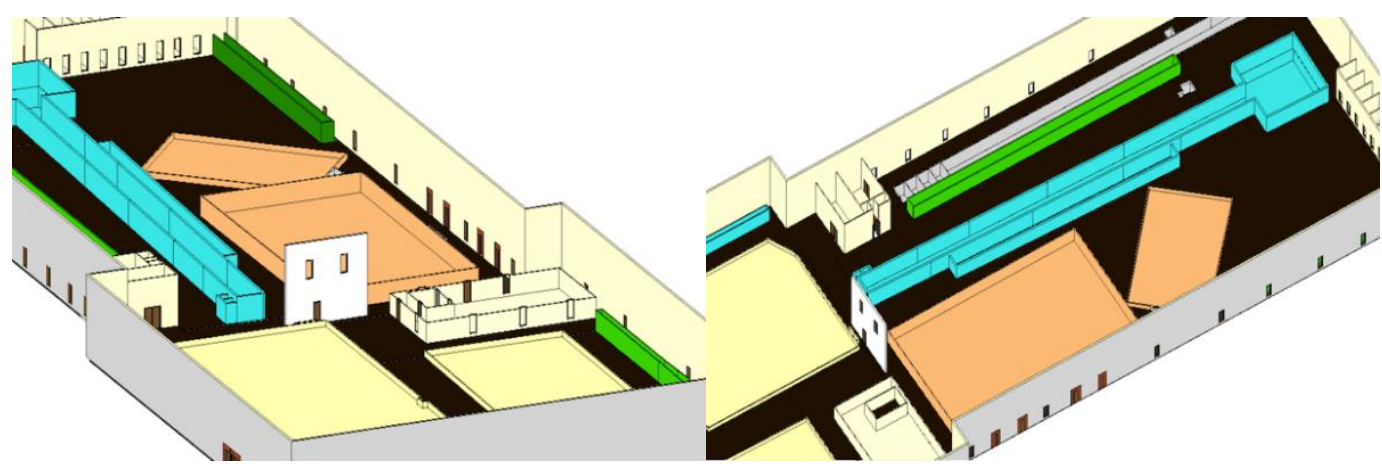

Figura 2: Modelo de Arquitetura da instalação.

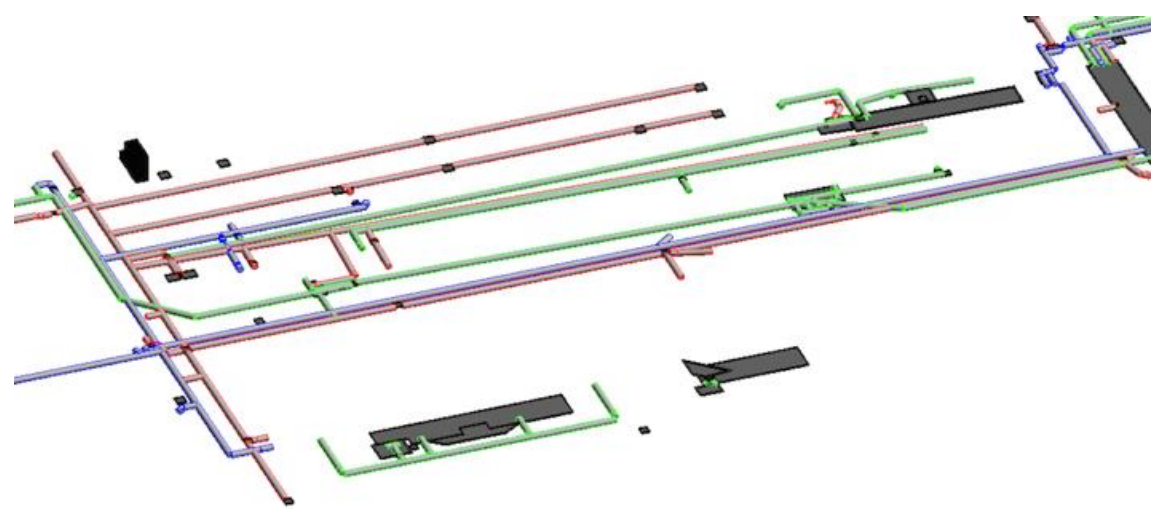

Figura 3: Modelo MEP. 
A Autodesk disponibiliza na página Autodesk - BIM Interoperability Tools, um Plug-in para fazer a extensão COBie (COBie Extension 2018) para o programa Revit 2018, de forma a facilitar a extração da informação contida nos modelos BIM para as folhas de cálculo COBie, sendo esta a opção escolhida para o trabalho realizado (uma outra alternativa seria recorrer ao formato IFC).

O Plug-in COBie Extension, é bastante simples de compreender e apresenta-se dividido em três separadores diferentes:

O primeiro separador corresponde ao Setup (Figura 4), ou seja, "Configuração", onde são introduzidas as informações relativamente aos contactos, e também a configuração de alguns parâmetros e o mapeamento de parâmetros.

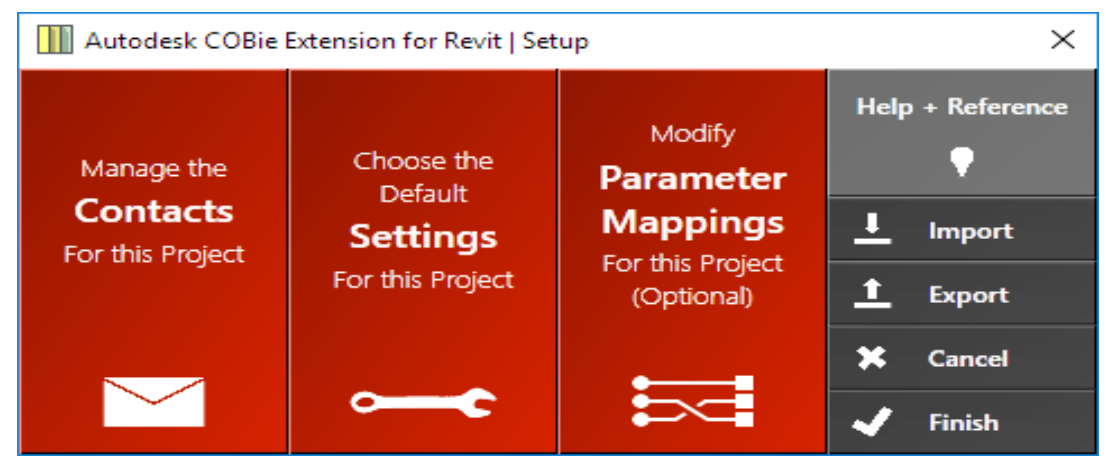

Figura 4: Separador SETUP do Autodesk COBie.

O segundo é o Modify (Figura 5), ou seja, "Modificação", onde se realizam as modificações para configurar a folha de cálculo, de forma a possibilitar as relações entre as zonas do modelo BIM e as zonas da especificação COBie. Nesta fase especificam-se as famílias, ou seja, os tipos de elementos que serão exportados.

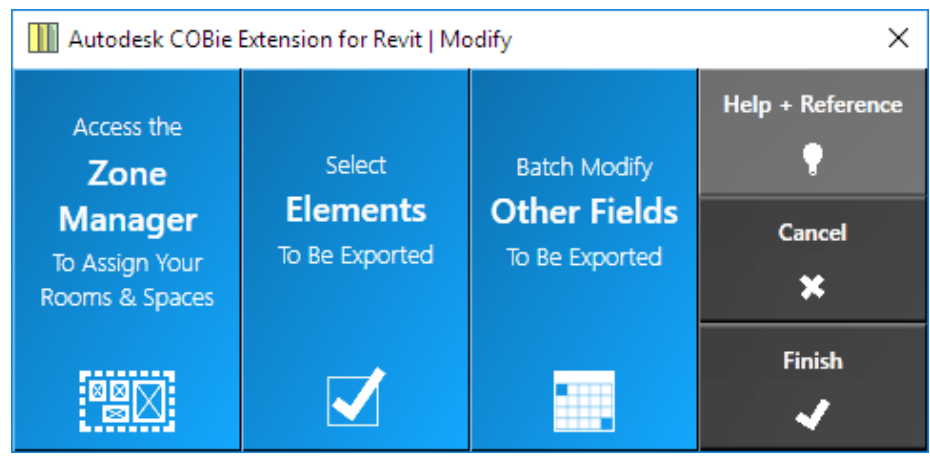

Figura 5: Separador MODIFY do Autodesk COBie.

O terceiro e último é o Export (Figura 6), ou seja, "Exportação", como o próprio nome indica corresponde à exportação e à criação do ficheiro COBie. Pretende-se configurar as folhas de cálculo a incluir, o formato do documento e a localização do ficheiro. 


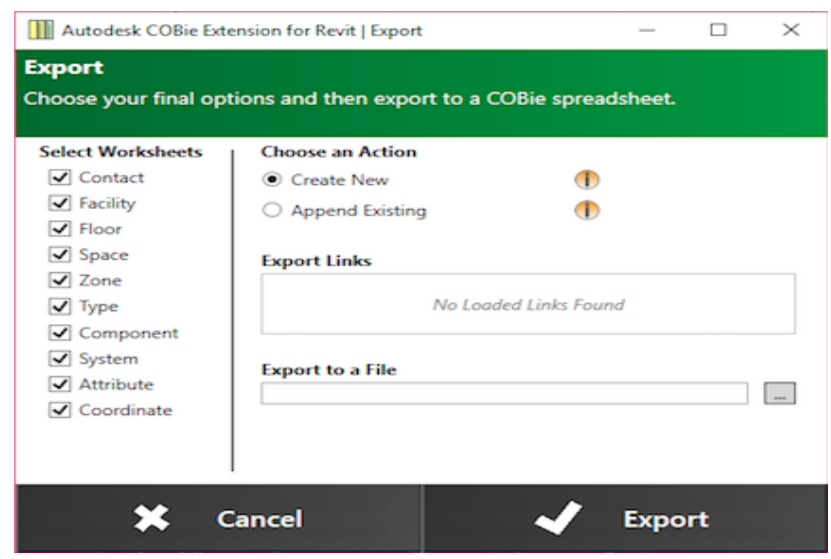

Figura 6: Separador EXPORT do Autodesk COBie.

O COBie inclui dezanove folhas de trabalho que são: contactos, documentos, ativos, pisos, tipos, espaços, componentes, zonas, sistemas, tarefas, recurso, reserva, atributos, coordenadas, edição e lista de seleção. A título de exemplo apresenta-se uma Folha de cálculo do COBie correspondente a uma Folha de Trabalho (Figura 7).

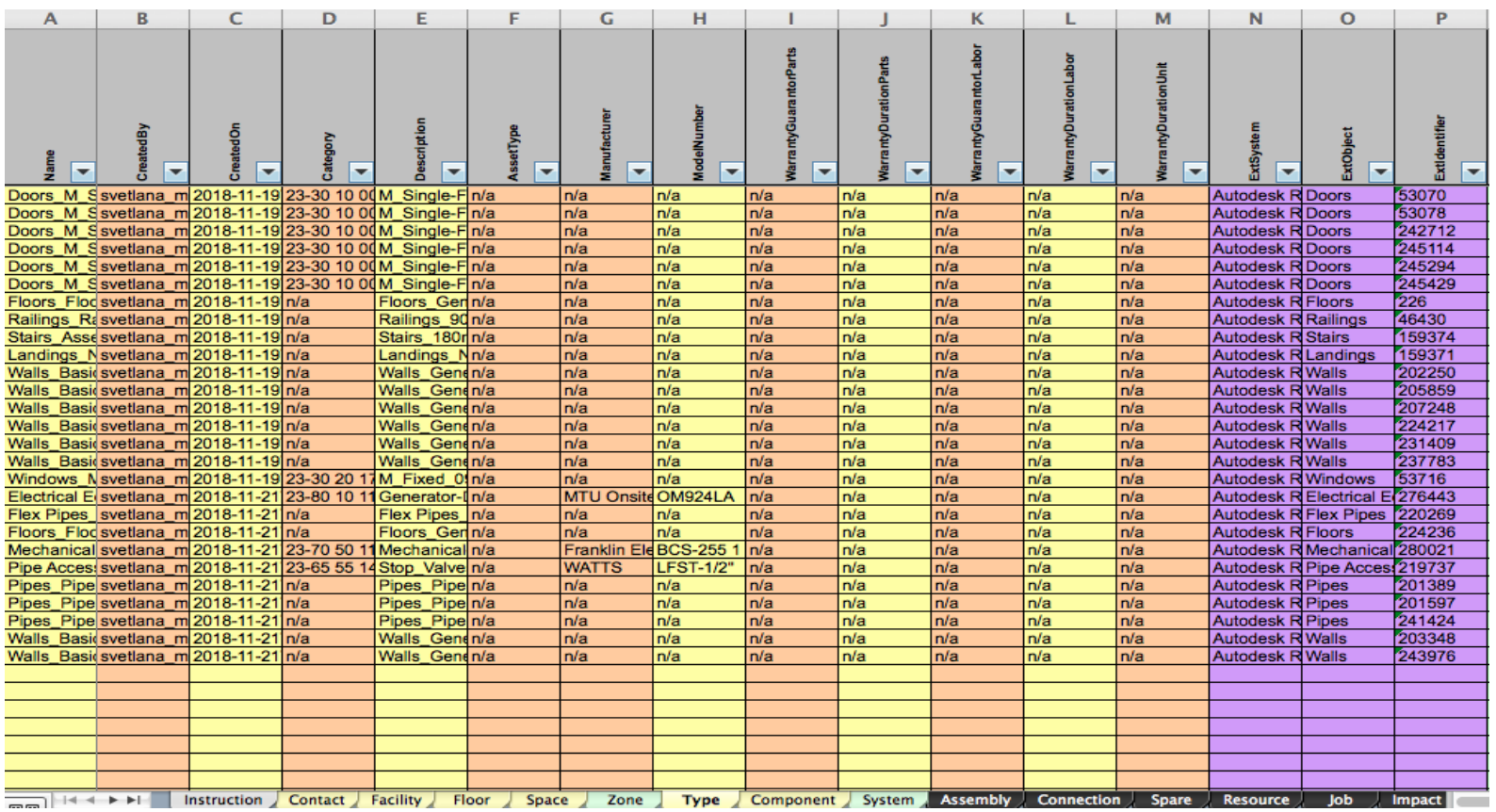

Figura 7: Exemplo da Folha de Cálculo "COBie" - "Folha de Trabalho Type do COBie".

Parte destas folhas de trabalho são preenchidas automaticamente pelo programa que fornece os dados COBie, enquanto a restante terá de ser preenchida manualmente. Existe uma primeira folha não editável designada instrução, onde é feita uma introdução à especificação COBie, contento as instruções necessárias para uma boa utilização das várias folhas de trabalho subsequentes e é dividida em três blocos (título, dados recolhidos em cada fase e código de cores). Relativamente ao código de cores indica-se: i) amarelo, informação requisitada pelo contrato; ii) laranja, informação de referência; iii) violeta, informação preenchida automaticamente pelo sistema; iv) verde, informação requisitada caso seja especificada; 
v) cinzento, informação secundária; vi) azul, para acrescentar informação complementar caso necessário; vii) preto, para assinalar folhas de trabalho não preenchidas por não terem sido solicitadas no contrato.

O primeiro passo paraa criação das folhas de cálculo COBie para a infraestrutura em estudo foi a edição das configurações gerais, com criação da lista dos contatos por forma a gerir, editar ou excluir os contatos dos envolvidos no projeto. Nesta lista, consta o nome, o e-mail, o telefone, o endereço, entre outros. Dentro dos contatos considerou-se a classificação da OmniClass com o código 34-20 1121 para Engenheiro Civil. Em seguida, noque se refere a configuração de parâmetros, foram editadas: i) Localização; ii) identificação, categoria-tipo e descriçãotipo; iii) espaços; iv) tipo; v) componentes; vi) sistemas; vii) atributos; viii) coordenadas; ix) horários. Posteriormente o Mapeamento de Parâmetros permitiu ignorar os parâmetros padrão da extensão COBie e usar os próprios parâmetros definidos no modelo BIM. Finalizase a configuração de parâmetros criando os schedule e os data nas propriedades de cada componente do modelo e também na informação do projeto. Num segundo passo, os dados COBie aplicados aos elementos durante a criação do modelo BIM fossem editados. Para a divisão dos espaços e zonas (Figura 1)no modelo foi tido em conta o seguinte: i) Todos os tanques, canais de ensaio e escritórios corresponderam a uma Room/space; ii) e as Zonas encontram-se divididas por 3 tipos diferentes (Zona A, Zona B e Zona C), em que cada uma delas agrupa diferentes tipos de Room/spaces.Após criação/definição, essas Zonas permitem mapeamento para qualquer uma das Roomspresentes no modelo elaborado. Foi também possível fazer seleção dos elementos e exportar (todas as famílias que se encontram no modelo) e ainda a correspondente visualização. Para finalizar geraram-se as folhas de cálculo COBie necessárias, como documentos do Microsoft Excel. Usando as configurações definidas nas etapas anteriores, neste procedimento especificaram-se quais as folhas de cálculo que seriam exportadas. Esta opção oferece a possibilidade de selecionar de entre as folhas de trabalho COBie as que realmente se pretende exportar.

\section{Conclusões ou Considerações Finais}

A principal dificuldade na modelação foi a falta de conformidade entre o modelo construído e o modelo em projeto. Na realidade, o modelo BIM desenvolvido para a Gestão de Ativos de uma construção existente tem que ter requisitos As-built, visto que a informação é toda exportada para as folhas COBie. Assim, devido à importância da utilização do nível de detalhe máximo na modelação, as informações do projeto tiveram que ser completadas in situ com os técnicos do laboratório. Para além do referido foi possível verificar-se também o acesso limitado a software de Gestão de Ativos, pois as licenças são bastante dispendiosas. Neste sentido não foi possível a exploração do desempenho total das folhas de cálculo COBie na utilização por software de Gestão de Ativos e a avaliação das dificuldades deste processo.

Conclui-se que os objetivos propostos foram alcançados, mesmo com as condicionantes identificadas no caminho percorrido, e que a Metodologia BIM aplicada à Gestão de Ativos, com base nas folhas de cálculo COBie é bastante vantajosa, pois permite a criação de uma base de dados que auxilia no processo da manutenção e operação dos ativos. A avaliação das folhas de cálculo COBie é bastante positiva, pois a informação o é armazenada, estruturada e extraída de um modelo BIM, e no processo da exportação cerca de metade da informação é preenchida automaticamente, o que evita erros ocorrentes da introdução manual da informação. 


\section{Referências}

[1] S. João, Metodologia BIM aplicada à gestão de ativos, Dissertação de Mestrado, Instituto Superior de Engenharia de Lisboa, Lisboa, Portugal, 2019.

[2] PAS 55-1, "Asset Management, Part 1 - Specification for the optimized management of physical assets", IAM - The Institute of Asset Management e BSI - British Standards Institution, 2018.

[3] PAS 55-2, "Asset Management, Part 2 - Guidelines for the application of PAS 55-1", IAM - The Institute of Asset Management e BSI - British Standards Institution, 2018.

[4] ISO 55000. (2016), International Organization for Standardization, “Asset management Overview, principles and terminology".

[5] ISO 55001. (2016), International Organization for Standardization, "Asset management Management systems - Requirements".

[6] ISO 55002. (2016), International Organization for Standardization, "Asset management Management systems" - Guidelines for the application of ISO 55001.

[7] C. Eastman, P. Teicholz, R. Sacks, K. Liston, BIM Handbook: A Guide to Building Information Modeling for Owners, Managers, Designers, Engineers, and Contractors (First). New Jersey, United States, 2008.

[8] D. Hamil, Building Information Modelling and Interoperability. NBS. UK, 2012.

[9] M. Martins, Aplicação do BIM a infraestrutura técnica: Sistema de Climatização. Instituto Superior de Engenharia de Lisboa. Lisboa, Portugal, 2017.

[10] R. Coelho, Aplicação do conceito de Gestão de Ativos Físicos numa Estação Elevatória de Águas. Instituto Superior de Engenharia de Lisboa, Engenharia Mecânica, 2015.

[11] J. Martins, O papel da engenharia na gestão de ativos de uma unidade industrial. Instituto Superior de Engenharia de Lisboa. Lisboa, Portugal, 2015.

[12] I. Rodas, Aplicação da metodologia BIM na Gestão de edifícios, Dissertação de Mestrado, Faculdade de Engenharia da Universidade do Porto, Porto, Portugal, 2015.

[13] NBS National BIM Report, 2014.

[14] Level of development specification, BIM Forum, 2015

[15] W. East, Construction Operations Building Information Exchange (COBIE): Requirements definition and pilot implementation standard. Final Report, Construction Engineering Research Laboratory (CERL), 2007.

[16] W. East, M. Carrasquillo-Mangual, The CoBie guide: A commentary to the NBIMS-US COBie standard, National Institute of Building Sciences, 2013 